\section{Admission or Observation? Population Health Is Here}

James A. Stewart, MD

It can be difficult enough to manage a patient's care when they have cancer that might require multidisciplinary approaches involving a variety of treatments and side effects. Increasingly, oncologists are asked to manage not just individuals but also populations of people, and to create systems that enhance efficiency and reduce cost. Accountable care organizations (ACOs) are being increasingly implemented, with the healthcare system being at financial risk and possibly deriving benefit based on overall expenditures and cost savings. Cancer tends to be a disease of older patients, and Medicare provides items and services for $>55$ million beneficiaries. ${ }^{1}$ Financial penalties are imposed when hospitals have an excess number of readmissions in a short period; patients with advanced cancer are at risk for this.

CMS (Centers for Medicare \& Medicaid Services) has provided special designations for patients who present to the emergency department (ED) with the opportunity for ED physicians and staff to designate patients as "observation status" or have them admitted to the inpatient service. If patients are in the hospital for $<2$ midnights, they will be listed as "observation" and not an "admission," and therefore not be at risk for the readmission penalty. Accurate designation can be tricky, and another layer of effort is required from the ED clinical staff.

My institution has a very large ED—one of the busiest in the northeast—and moving patients with a wide acuity of problems safely through the system can be challenging in the best of times. For our large hospital, the financial risks related to poor management of observation status designation is such that our case management group actually assigned specific staff to keep track of observation status patients and optimally manage the situation. Interestingly, our senior case manager said that she believes in the future the system will move beyond the "observation" label toward a more "universal medicine" approach in which accountable care principles will address populations across the outpatient and inpatient spectrum. Certainly many healthcare systems remain "hospital-centric," even to the point of maintaining smaller financially challenged hospitals that cannot really provide the depth of expertise available at larger centers.

Oncologists have known for years that cancer care is primarily an outpatient activity. Decades ago, we moved chemotherapy from the inpatient to the outpatient setting and continue to work with outstanding oncology nurses who help keep patients out of the hospital.

Why do patients with cancer go to the ED? Mayer et al ${ }^{2}$ used a state-wide database to assess this question in North Carolina. They found that the top 3 complaints that led to an ED visit were related to pain, respiratory distress, and gastrointestinal issues. Lung cancer was the most common cancer in this population, and patients with lung cancer were the most likely to be admitted. A more recent analysis of the literature by Lash et $\mathrm{al}^{3}$ found that ED use among patients with cancer exceeded that of the general population. Again, the types of symptoms leading to an ED visit would be familiar to all oncologists (chemotherapy and surgical complications, pain, respiratory symptoms, and gastrointestinal symptoms).

In this issue of JNCCN, Lipitz-Snyderman et al (page 1234) provide a thoughtful study related to the observation designation in older patients with cancer who present to the ED. They used population-based SEER-Medicare data with $>150,000$ patients per cohort. The focus was on patients with breast, colon, lung, and prostate cancers. Observation status was used less frequently in patients with cancer compared with those without (43 vs 69 observation status visits per 1,000 inpatient admissions, respectively).

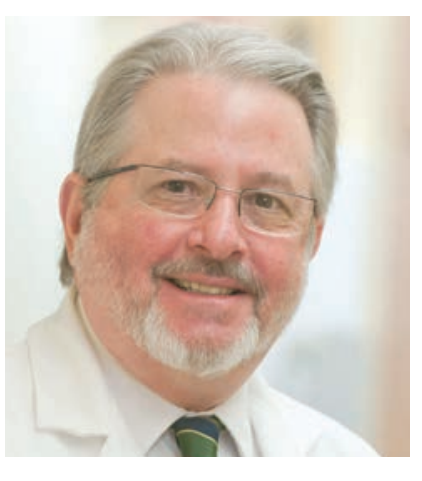

James A. Stewart, MD

James A. Stewart, MD, is a medical oncologist and the Division Chief, Hematology-Oncology at Baystate Medical Center in Springfield, Massachusetts. His clinical focus is in breast cancer and he has over 25 years of experience as a fellowship director. 
The authors provide data that were available to them in terms of cancer stage, geographic region, and age, for example. Although beyond the scope of their project, it would be interesting to know how much communication occurred before patients arrived at the ED. For example, what efforts were made by the treating cancer program to keep the patient out of the ED? Were the patients admitted from home or clinic? What percentage of patients had clear-cut advanced directives? These are all questions we will need to address as we build our skill sets in managing populations in addition to individuals.

In 1979, I spent several months in Glasgow, Scotland, working in a geriatric unit. Visiting nurses did extraordinary work keeping people out of acute care hospitals. When they had questions about the need for hospitalization, physicians would make a home visit before any decision. This is a model we should be using more. I expect we will need to mobilize the appropriate resources to do more home care and outpatient work for our cancer populations. Healthcare organizations can no longer afford to be dominantly hospital centric.

Many oncology physicians struggle with when to forego anticancer treatment and move to a best supportive care approach. Grudzen et $\mathrm{al}^{4}$ describe a randomized clinical trial comparing palliative care initiated in the ED versus usual care. They concluded that this approach improves quality of life in patients with advanced cancer but does not shorten survival. Innovative approaches such as this are needed to enhance the overall process of reducing unnecessary hospitalizations.

Management of populations will become the norm in cancer care. On-call physicians will need to have efficient pathways for patients needing increased intensity of care. Too often the path of least resistance is to go to the ED and, once there, be admitted. I expect it will be useful for all cancer programs of medium to large size to create algorithms jointly with the ED staff for enhanced patient flow depending on the clinical scenario. Likewise, for programs such as ours where there is a very large hospitalist staff, they will need support in getting patients back to the clinic quickly and not use valuable hospital resources excessively.

I would encourage leadership in any cancer program to review the paper by LipitzSnyderman et al. It raises a number of questions that we will have to answer as Medicare and other payors increasingly promote the idea of efficient population management.

\section{References}

1. Centers for Medicare \& Medicaid Services. Medicare Coverage Center. Available at: https://www.cms.gov/Center/ Special-Topic/Medicare-Coverage-Center.html. Accessed September 19, 2017.

2. Mayer DK, Travers D, Wyss A, et al. Why do patients with cancer visit emergency department? Results of a 2008 population study in North Carolina. J Clin Oncol 2011;29:2683-2688.

3. Lash RS, Bell JF, Reed SC, et al. A systematic review of emergency department use among cancer patients. Cancer Nurs 2017;40:135-144.

4. Grudzen CR, Richardson LD, Johnson PN, et al. Emergency department-initiated palliative care in advanced cancer: a randomized clinical trial. JAMA Oncol 2016;2:591-598. 